Artículo original

\title{
Abandono del tratamiento en tuberculosis multirresistente: factores asociados en una región con alta carga de la enfermedad en Perú
}

\author{
Oriana Rivera ${ }^{1,2}$, Santiago Benites ${ }^{1}$, Julio Mendigure ${ }^{2}$, César Antonio Bonilla ${ }^{2,3}$ \\ ${ }^{1}$ Vicerrectorado de Investigación, Universidad César Vallejo, Lima, Perú \\ ${ }^{2}$ Facultad de Ciencias de la Salud, Universidad Norbert Wiener, Lima, Perú \\ ${ }^{3}$ Oficina de Apoyo a la Docencia e Investigación, Hospital Nacional Daniel Alcides Carrión, \\ Callao, Perú
}

Introducción. En la tuberculosis multirresistente, el abandono del tratamiento constituye un grave problema de salud pública que afecta la calidad de vida de los pacientes, sus familias y la comunidad. El enfrentarlo supone una carga para los sistemas sanitarios debido a que provoca fuentes de transmisión libre en la comunidad e incrementa la prevalencia y la mortalidad. De ahí, la necesidad de investigar los factores asociados con esta situación. Objetivo. Determinar los factores de riesgo asociados con el abandono del tratamiento en pacientes con tuberculosis multirresistente en la región de Callao (Perú).

Materiales y métodos. Se hizo un estudio analítico de casos y controles (80 casos y 180 controles) en tratamiento entre el $1^{\circ}$ enero del 2010 y el 31 diciembre del 2012. Los factores se determinaron mediante regresión logística, y se calcularon los odds ratios (OR) y los intervalos de confianza (IC) del $95 \%$.

Resultados. En el análisis multivariado se determinaron los siguientes factores de riesgo: no tener conocimiento de la enfermedad $\left(\mathrm{OR}=23,10 ; \mathrm{IC}_{95 \%}: 3,6-36,79 ; \mathrm{p}=0,002\right)$; no creer en la curación $\left(O R=117,34 ; I_{95 \%}: 13,57-124,6 ; p=0,000\right)$; no tener apoyo social $\left(\mathrm{OR}=19,16 ; \mathrm{IC}_{95 \%}: 1,32-27,77 ; \mathrm{p}=0,030\right)$; no considerar adecuado el horario de atención $\left(\mathrm{OR}=78,13 ; \mathrm{IC}_{95 \%}: 4,84-125,97 ; \mathrm{p}=0,002\right)$, y no recibir los resultados de laboratorio $\left(\mathrm{OR}=46,13 ; \mathrm{IC}_{95 \%}: 2,85-74,77 ; \mathrm{p}=0,007\right)$.

Conclusión. Los servicios de salud deben esforzarse en la determinación precoz de las condiciones que podrían convertirse en factores de riesgo, lo cual ayudaría a implementar preventivamente intervenciones efectivas, rápidas y de alto impacto.

Palabras clave: tuberculosis; resistencia a medicamentos; factores de riesgo.

Recibido: 08/06/18 Aceptado: 02/11/18 Publicado: 12/12/18

Citación:

Rivera O, Benites S, Mendigure J, Bonilla CA. Abandono del tratamiento en tuberculosis multirresistente: factores asociados en una región con alta carga de la enfermedad en Perú. Biomédica. 2019;39(Supl.2):44-57.

https://doi.org/10.7705/biomedica.v39i3.4564

Correspondencia:

César Antonio Bonilla, Oficina de Apoyo a la Docencia e Investigación, Hospital Nacional Daniel Alcides Carrión, Av. Guardia Chalaca 2176, Callao, Perú

Teléfono: (51) 6147474; extensión 3312

bonasal@gmail.com

Contribución de los autores:

Todos los autores participaron en la preparación del proyecto, el diseño, la recolección de la muestra, el análisis de resultados y la redacción del manuscrito.

Financiación:

Esta investigación fue financiada con recursos propios de los investigadores.

Conflicto de intereses:

Los autores declaramos no tener conflictos de intereses.

\section{Abandonment of therapy in multidrug-resistant tuberculosis: Associated factors in a region with a high burden of the disease in Perú}

Introduction: In the context of multidrug-resistant tuberculosis, abandonment of therapy represents a serious public health problem that affects the quality of life of patients, families, and communities. Managing this phenomenon places a burden on health systems since it causes free sources of transmission in the community, thereby increasing prevalence and mortality. Thus, there is a need to study factors associated with this problem.

Objective: This study sought to identify risk factors associated with the abandonment of therapy by patients with multidrug-resistant tuberculosis in the Peruvian region of Callao. Materials and methods: We conducted an analytical case-control study (cases $=80$; controls $=180$ ) in patients under treatment from January $1^{\text {st }}, 2010$, to December 31, 2012. Risk factors were identified using logistic regression; odds ratios (OR) and $95 \%$ confidence intervals $(\mathrm{Cl})$ were calculated.

Results: The multivariate analysis identified the following risk factors: Being unaware of the disease (OR=23.10; 95\% Cl 3.6-36.79; $p=0.002)$; not believing in healing (OR=117.34; 95\% $\mathrm{Cl} 13.57-124.6 ; p=0.000)$; not having social support (OR=19.16; 95\% $\mathrm{Cl} 1.32-27.77 ; p=0.030)$; considering the hours of attention to be inadequate $(\mathrm{OR}=78.13 ; 95 \% \mathrm{Cl} 4.84-125.97 ; p=0.002)$, and not receiving laboratory reports (OR=46.13; 95\% $\mathrm{Cl} 2.85-74.77 ; \mathrm{p}=0.007)$.

Conclusion: Health services must focus on the early detection of conditions that may represent risk factors to proactively implement effective, rapid and high-impact interventions.

Keywords: Tuberculosis, drug resistance, risk factor.

En la actualidad, los programas de control de la tuberculosis en el mundo tienen como reto principal conseguir la observancia del tratamiento. Se ha demostrado que, cuando se cumple estrictamente el esquema terapéutico, este resulta ser de gran eficacia para tratar las formas sensibles $y$ resistentes de la enfermedad. El enfrentar la irregularidad y el abandono del 
tratamiento supone una carga adicional para los sistemas sanitarios porque provocan fuentes de transmisión de tuberculosis libre en la comunidad, lo que contribuye a incrementar la prevalencia y la mortalidad a pesar de los grandes avances en el diagnóstico y el tratamiento.

La tuberculosis multirresistente (TB-MDR), forma clínica y bacteriológica grave de la enfermedad, es ocasionada por una cepa de Mycobacterium tuberculosis resistente, por lo menos, a la isoniacida y a la rifampicina, medicamentos esenciales en los esquemas de tratamiento de primera línea. Se ha reportado un deficiente cumplimiento del tratamiento farmacológico a nivel global, lo que configura una situación verdaderamente crítica, cuya expansión debe evitarse.

La Organización Mundial de la Salud (OMS) registró 600.000 nuevos casos de tuberculosis resistente a la rifampicina en el mundo durante el 2016; de ellos, 490.000 eran multirresistentes, el $50 \%$ se concentraba en tres países: India, China y Rusia, y el éxito del tratamiento se logró únicamente en el $54 \%$ (1). Perú es uno de los países de Latinoamérica con mayor cantidad de casos de tuberculosis resistente a la rifampicina o multirresistente; en ese mismo año, 2016, se estimaron en 2.300 los casos, 1.645 de los cuales iniciaron el tratamiento en el periodo considerado. Los resultados preliminares de la cohorte de tratamiento, indicaron que la tasa de éxito al finalizar el estudio no sería mayor del $52 \%(1,2)$ debido, principalmente, al abandono del tratamiento que, históricamente, es de alrededor del $30 \%$ (3).

La tuberculosis es una enfermedad que resulta de la combinación de múltiples factores, tales como variaciones demográficas y ajustes territoriales, falta de recursos básicos, y bajos ingresos personales y familiares, lo que implica migraciones, hacinamiento y desnutrición, entre otros. A ello se suman estilos de vida inadecuados, comorbilidades como el HIV/sida, la diabetes mellitus, el cáncer y otras, además del deterioro en las intervenciones de salud pública determinado por la debilidad de los equipos asistenciales y del mismo sistema de salud, lo cual impide el diagnóstico precoz, el inicio oportuno del tratamiento y su cumplimiento. Estas situaciones contribuyen a la adaptación de M. tuberculosis y favorecen el desarrollo de la resistencia a los antibióticos existentes $(4,5)$.

En el control de la tuberculosis sensible y de sus formas resistentes, la falta de fidelidad al servicio de salud y de cumplimiento del esquema terapéutico, constituyen un grave problema de salud pública que puede afectar la calidad de vida de las personas afectadas, de sus familias y de la comunidad, y ocasionar costos innecesarios para el sistema de salud e incrementar el gasto de las familias; además, la ausencia de resultados clínicos, epidemiológicos y sociales favorables constituye una barrera para el control de la enfermedad $(6,7)$.

El abandono del tratamiento antituberculoso responde a muchas causas. Se han evaluado factores sociodemográficos como el sexo, la edad, la ocupación, el alcoholismo y la adicción a drogas ilegales, así como factores relacionados con las condiciones de acceso a la atención médica de los pacientes y a la organización en la prestación de los servicios de salud $(8,9)$.

El propósito del estudio fue identificar, entender, explicar, valorar y vincular entre sí los factores de riesgo asociados con el abandono del tratamiento de pacientes con tuberculosis multirresistente, con el fin de caracterizar el perfil de los afectados para, así, reforzar conocimientos, mejorar actitudes y promover prácticas y estilos de vida encaminadas a la prevención y a fomentar el cumplimiento del tratamiento. 


\section{Materiales y métodos}

Se hizo un estudio de diseño observacional, analítico, de casos y controles, no pareado. La población de interés fueron las personas con tuberculosis multirresistente que iniciaron el tratamiento en los establecimientos de la Dirección Regional de Salud del Callao (Perú) bajo condiciones de supervisión y gratuidad en el periodo comprendido entre el $1^{\circ}$ de enero del 2010 y el 31 de diciembre del 2012, y que para el 31 de julio de 2015 se consideraron 'curados' o habían 'abandonado' el tratamiento según las normas técnicas para el manejo diagnóstico y terapéutico de la tuberculosis multirresistente en el país (10).

Se contempló el uso de tres tipos de tratamiento:

i. estandarizado, es decir, el prescrito para aquellos con factores de riesgo para la tuberculosis multirresistente y quienes, por la gravedad de su estado, no podían esperar el resultado de una prueba de sensibilidad rápida o convencional para iniciar el tratamiento consistente en 6 a 8 meses de etambutol, pirazinamida, levofloxacina, kanamicina, etionamida, cicloserina, o 12 a 16 meses de etambutol, pirazinamida, levofloxacina, etionamida, cicloserina;

ii. empírico, o sea, el administrado a personas con diagnóstico de tuberculosis resistente y con resultados en cualquiera de las dos pruebas de sensibilidad rápida utilizadas en el país (Microscopic Observation Drug Susceptibility, MODS, o la prueba molecular Genotype MTBDR plus ${ }^{\mathrm{TM}}$ ), o la prueba de sensibilidad convencional a fármacos de primera y segunda línea (método de proporciones en agar en placa), así como a contactos domiciliarios de un caso de tuberculosis multirresistente en quienes el esquema se basa en el tratamiento del caso índice, e

iii. individualizado, es decir, el que se basa en los resultados de una prueba de sensibilidad convencional a los medicamentos de primera y segunda línea.

Se definieron como "casos" a aquellas personas que recibieron tratamiento para tuberculosis multirresistente y cuya condición de egreso se registró como abandono del tratamiento (paciente que inicia el tratamiento y lo suspende durante 30 días consecutivos o más). Como "controles", se incluyeron aquellas personas que recibieron tratamiento para la tuberculosis multirresistente y que al egreso se registraron como curadas (pacientes que concluyeron el esquema de tratamiento y tuvieron resultados negativos en diez cultivos durante los doce meses anteriores).

En el periodo de investigación, se notificaron 408 personas con tuberculosis multirresistente que iniciaron el tratamiento en la región de Callao; de ellos, 86 cumplían con los criterios de inclusión para ser considerados como casos y 180 pacientes cumplían con la definición y los criterios de inclusión para ser considerados como controles (figura 1). Los casos y controles acudieron espontáneamente al Hospital Nacional Daniel Alcides Carrión o fueron remitidos desde los establecimientos de salud de la Dirección de Salud de Callao.

Tanto los casos como los controles tuvieron representatividad, simultaneidad y homogeneidad, y la selección se basó en los siguientes criterios de inclusión: pacientes mayores de 18 años con resultados para la prueba de sensibilidad a medicamentos de primera y segunda línea tomada al inicio del tratamiento. Se excluyeron aquellos pacientes con información incompleta en la historia clínica.

La recolección de los datos se hizo a partir de una ficha de recolección de datos validada por expertos en tuberculosis y en metodología de la investigación. 


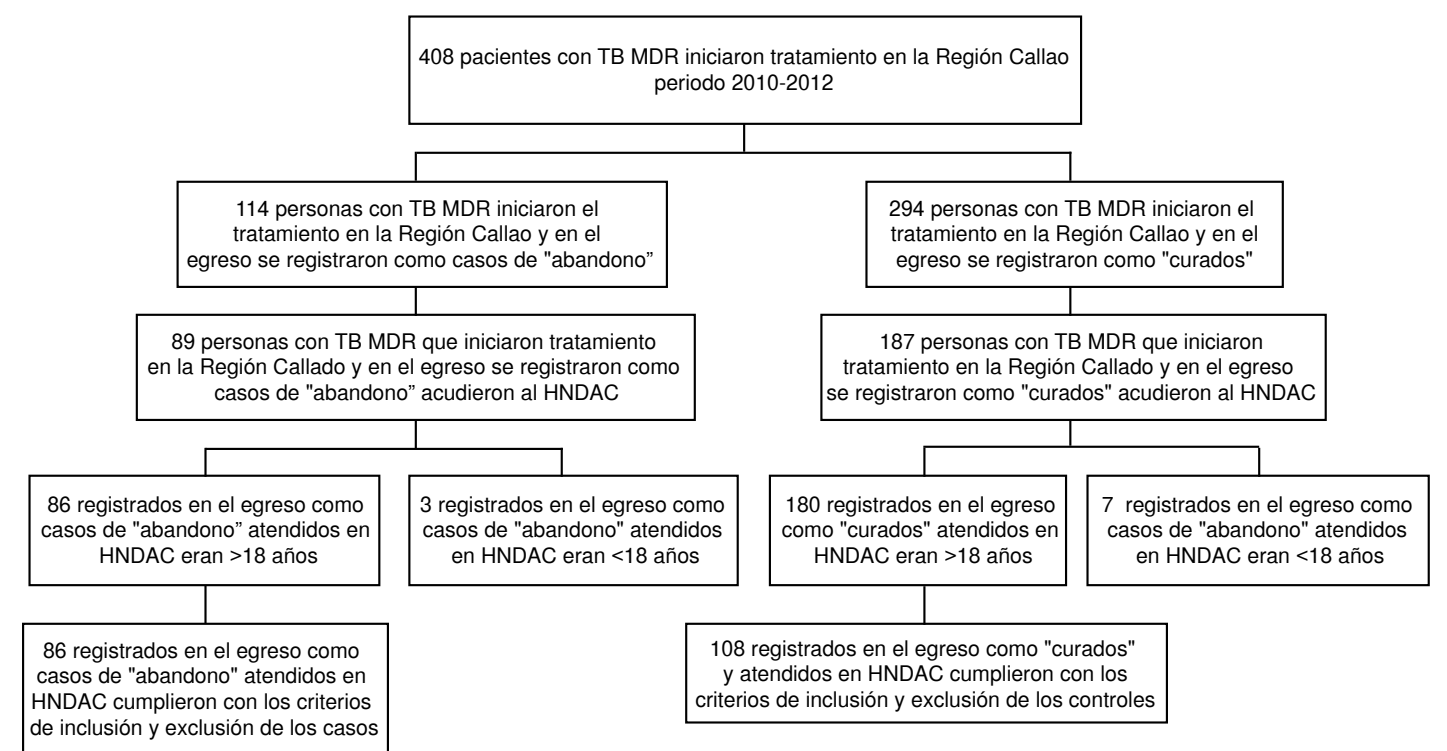

Figura 1. Flujograma de la selección de individuos para el estudio HNDAC: Hospital Nacional Daniel Alcides Carrión

Las variables explicativas de los factores de riesgo para el abandono del tratamiento se definieron de acuerdo con cuatro dimensiones con sus respectivos indicadores: biología humana (sexo, edad, antecedentes de hospitalización, reacciones adversas a medicamentos antituberculosos, mejoría durante el tratamiento); ambiente (estado civil, escolaridad, ocupación, ingresos económicos familiares, vivienda donde residió durante el tratamiento, distancia al establecimiento de salud, conocimiento sobre la enfermedad, creencias sobre la curación, apoyo familiar y apoyo social); estilos de vida (consumo de drogas alucinógenas, alcohol y tabaco), y servicios de salud (relación con el personal de salud, explicación sobre la enfermedad y el tratamiento, horario de atención y tiempo de espera para la administración del tratamiento, no poder seguir el tratamiento por falta de personal y medicamentos, comodidad, información sobre los resultados de laboratorio, atención ambulatoria oportuna, y visita domiciliaria cuando dejó de recibir el tratamiento).

Además, se consideraron indicadores como el tiempo y el tipo de tratamiento, el retorno al establecimiento luego del abandono, la confirmación diagnóstica de la tuberculosis multirresistente y la conversión bacteriológica al sexto mes de tratamiento.

Se hizo un análisis exploratorio inicial de los datos para determinar la frecuencia de presentación de cada variable y su distribución. En las variables continuas se obtuvieron medidas de tendencia central (medias, medianas) y de dispersión, según la naturaleza de los datos. El proceso de descripción de las variables categóricas se complementó con tablas de frecuencia.

Posteriormente, se hizo un análisis bivariado. La asociación estadística entre las variables de resultado y las explicativas categóricas se evaluó mediante tablas de contingencia, empleando la razón de disparidad (odds ratio, OR) con su correspondiente intervalo de confianza (IC) del $95 \%$. La significación estadística de las tablas de contingencia se evaluó mediante la prueba de ji al cuadrado o la prueba exacta de Fisher cuando fue necesario. A continuación, se hizo un análisis de regresión logística múltiple que permitió determinar aquellas variables que explicaron el modelo final. 


\section{Consideraciones éticas}

El estudio contó con la aprobación del Comité de Ética del Hospital Nacional Daniel Alcides Carrión de Callao (Perú).

\section{Resultados}

En el estudio se incluyeron 266 personas (86 casos y 180 controles), el $60,2 \%$ correspondía a hombres, el $85 \%$ tenía entre 18 y 47 años de edad, el $62,4 \%$ tenía educación primaria, el 55,3 \% mantenía una relación de pareja (23,3\%, convivientes y $32 \%$, casados), el $15 \%$ estaba desocupado y el $27 \%$ estudiaba. El 86,1\% tenía un ingreso económico familiar de hasta USD\$ 465 mensuales, y el 55,6 \% percibía hasta USD\$232. El 28,6\% de los pacientes recibió 12 meses de tratamiento en promedio.

Según el estudio, el 68 \% de los casos respondió no haber retornado al establecimiento de salud donde recibía el tratamiento luego de abandonarlo. En cuanto al tiempo de tratamiento en el momento del abandono, el $60 \%$ lo hizo a los seis meses y, el $35 \%$, antes del año. El $72 \%$ de los abandonos se dio en personas que recibían el esquema estandarizado según la norma nacional. El $30 \%$ no tenía confirmación diagnóstica de multirresistencia en el momento del abandono y, un dato bastante interesante, 49 de los casos que abandonaron el tratamiento lo hicieron después de seis meses de iniciado, y el $71 \%$ de quienes abandonaron el tratamiento tenía conversión bacteriológica al sexto mes de tratamiento.

En el análisis bivariado se observó que los factores asociados con el abandono en lo que se refiere a la dimensión 'biología humana' fueron: edad entre 28 y 37 años, haber estado hospitalizado de uno a tres meses al inicio del tratamiento, no sentir mejoría durante el tratamiento y tener diabetes mellitus (cuadro 1).

Cuadro 1. Características de la población según la dimensión 'biología humana'

\begin{tabular}{|c|c|c|c|c|}
\hline Características & $\begin{array}{c}\text { Casos }(n=86) \\
n(\%)\end{array}$ & $\begin{array}{c}\text { Controles }(n=180) \\
n \quad(\%)\end{array}$ & OR IC $_{95 \%}$ & $\mathbf{p}$ \\
\hline \multicolumn{5}{|l|}{ Sexo } \\
\hline Masculino & $57(66,3)$ & $103(57,2)$ & $1,46 \quad(0,86-2,51)$ & 0,159 \\
\hline Femenino & $29(33,7)$ & $77(42,8)$ & & \\
\hline \multicolumn{5}{|l|}{ Edad (años) } \\
\hline 18 a 27 & $29(33,7)$ & $82(45,6)$ & $0,60 \quad(0,35-0,03)$ & 0,044 \\
\hline 28 a 37 & $33(38,4)$ & $39(21,7)$ & $2,25 \quad(1,28-3,94)$ & 0,004 \\
\hline 38 a 47 & $15(17,4)$ & $28(15,6)$ & $1,14 \quad(0,57-2,28)$ & 0,411 \\
\hline 48 o más & $9(10,5)$ & $31(17,2)$ & 1 & \\
\hline \multicolumn{5}{|l|}{ Tiempo de hospitalización (días) } \\
\hline$<14$ & $4(10,3)$ & $36(55,4)$ & 1 & \\
\hline 14 a 30 & $17(43,6)$ & $27(41,5)$ & $0,92 \quad(0,41-2,05)$ & 0,838 \\
\hline 30 a 90 & $13(33,3)$ & $2(3,1)$ & $5,30(1,45-19,42)$ & 0,000 \\
\hline \multicolumn{5}{|l|}{$\begin{array}{l}\text { Durante el tratamiento, ¿sintió } \\
\text { mejoría en el estado de salud? }\end{array}$} \\
\hline No & $60(69,8)$ & $53(29,4)$ & $5,53 \quad(3,15-9,68)$ & 0,000 \\
\hline Sí & $26(30,2)$ & $127(70,6)$ & & \\
\hline \multicolumn{5}{|l|}{ Otra enfermedad diagnosticada } \\
\hline Asma & $10(20,8)$ & $12(27,3)$ & 1 & \\
\hline HIV & $10(20,8)$ & $3(6,8)$ & $0,27 \quad(0,07-1,08)$ & 0,050 \\
\hline Diabetes & $23(47,9)$ & $10(22,7)$ & $6,20(2,79-13,76)$ & 0,000 \\
\hline Insuficiencia renal crónica & $3(6,2)$ & $9(20,5)$ & $0,68 \quad(0,18-2,60)$ & 0,419 \\
\hline Enfermedad hepática crónica & $1(2,1)$ & $1(2,3)$ & $2,10(0,13-34,07)$ & 0,543 \\
\hline Cáncer & $1 \quad(2,1)$ & $5(11,4)$ & $6,02(0,67-53,76)$ & 0,083 \\
\hline
\end{tabular}

OR: Odds ratio 
En cuanto a la dimensión 'ambiente', los factores fueron tener un nivel de instrucción secundario, un ingreso inferior a USD\$232 mensuales, viajar más de una hora para llegar al establecimiento de salud, falta de conocimiento sobre la enfermedad o no contar con apoyo familiar o social (cuadro 2).

Cuadro 2. Características de la población según la dimensión 'ambiente'

\begin{tabular}{|c|c|c|c|c|}
\hline Características & $\begin{array}{c}\text { Casos }(n=86) \\
n(\%)\end{array}$ & $\begin{array}{c}\text { Controles }(n=180) \\
n(\%)\end{array}$ & OR IC ${ }_{95 \%}$ & $\mathbf{p}$ \\
\hline \multicolumn{5}{|l|}{ Estado civil } \\
\hline Viudo & $1(1,2)$ & $1(0,6)$ & 1 & \\
\hline Soltero & $28(32,6)$ & $89(49,4)$ & $0,49 \quad(0,28-0,84)$ & 0,007 \\
\hline Conviviente & $25(29,1)$ & $37(20,6)$ & $1,58 \quad(0,87-2,85)$ & 0,085 \\
\hline Casado & $32(37,2)$ & $53(29,4)$ & $1,42 \quad(0,82-2,44)$ & 0,130 \\
\hline \multicolumn{5}{|l|}{ Escolaridad } \\
\hline Nunca asistió a la escuela & $6(7,0)$ & $3(1,7)$ & 1 & \\
\hline Primaria & $61(70,9)$ & $105(58,3)$ & $3,44(0,83-14,26)$ & 0,088 \\
\hline Secundaria & $17(19,8)$ & $68(37,8)$ & $8,00(1,81-35,29)$ & 0,006 \\
\hline Superior & $2(2,3)$ & $4(2,2)$ & $4,00(0,44-35,77)$ & 0,215 \\
\hline \multicolumn{5}{|l|}{$\begin{array}{l}\text { Distancia al establecimiento } \\
\text { de salud (minutos) }\end{array}$} \\
\hline$<15$ & $10(11,6)$ & $28(15,6)$ & 1 & \\
\hline $15-30$ & $18(20,9)$ & $52(28,9)$ & $1,03 \quad(0,42-2,53)$ & 0,946 \\
\hline $31-60$ & $42(48,8)$ & $90(50,0)$ & $0,76 \quad(0,34-1,72)$ & 0,517 \\
\hline$>60$ & $16(18,6)$ & $10(5,6)$ & $1,84 \quad(1,12-3,01)$ & 0,001 \\
\hline \multicolumn{5}{|l|}{$\begin{array}{l}\text { Conocimiento sobre la } \\
\text { enfermedad }\end{array}$} \\
\hline No & $74(86,0)$ & $135(75,0)$ & $2,05 \quad(1,02-4,12)$ & 0,027 \\
\hline Sí & $12(14,0)$ & $45(25,0)$ & & \\
\hline \multicolumn{5}{|l|}{ Creencias sobre la curación } \\
\hline No & $73(84,9)$ & $41(22,8)$ & $19,10(9,59-37,77)$ & 0,000 \\
\hline Sí & $13(15,1)$ & $139(77,2)$ & & \\
\hline \multicolumn{5}{|l|}{ Apoyo familiar } \\
\hline No & $42(48,8)$ & $18(10,0)$ & $8,59(4,50-16,37)$ & 0,000 \\
\hline Sí & $44(51,2)$ & $162(90,0)$ & & \\
\hline \multicolumn{5}{|l|}{ Apoyo social } \\
\hline No & $42(48,8)$ & $19(10,6)$ & $8,08(4,28-15,28)$ & 0,000 \\
\hline Sí & $44(51,2)$ & $161(89,4)$ & & \\
\hline
\end{tabular}

En el ámbito de los 'estilos de vida', se encontraron factores de riesgo como el consumo de drogas alucinógenas y el de tres a cuatro cigarrillos al día (cuadro 3).

En relación con los servicios de salud, se encontró que la mala relación con el personal de salud, no recibir explicación sobre la enfermedad, no estar de acuerdo con los horarios de atención ni los tiempos de espera para la atención y un ambiente poco cómodo, fueron los factores de riesgo para abandonar el tratamiento (cuadro 4).

El análisis multivariado confirmó que los factores asociados con el abandono del tratamiento fueron: no tener conocimiento sobre la enfermedad $\left(\mathrm{OR}=23,10 ; \mathrm{IC}_{95 \%}: 3,96-36,79 ; \mathrm{p}=0,02\right)$, creer que no se iba a obtener la cura $\left(O R=117,34: I_{95 \%} 13,57-124,6 ; p=0,00\right)$, no contar con apoyo social $\left(\mathrm{OR}=19,16 ; \mathrm{IC}_{95 \%}: 1,32-27,77 ; \mathrm{p}=0,03\right)$, no considerar adecuado el horario del establecimiento de salud (OR=78,13; $\left.I C_{95 \%}: 4,84-125,97 ; p=0,02\right)$ y que el personal de salud no informara sobre los resultados de laboratorio 
Cuadro 3. Características de la población según la dimensión 'estilos de vida'

\begin{tabular}{lcrrr}
\hline Características & $\begin{array}{c}\text { Casos (n=86) } \\
\mathbf{n ~ ( \% )}\end{array}$ & $\begin{array}{c}\text { Controles (n=180) } \\
\mathbf{n ~ ( \% )}\end{array}$ & OR IC $_{95 \%}$ & p \\
\hline $\begin{array}{l}\text { Durante el tratamiento, ¿ingirió } \\
\text { bebidas que contenían alcohol? }\end{array}$ & $41(47,7)$ & $156(88,7)$ & $0,14(0,07-0,25)$ & 0,000 \\
$\quad$ No & $45(52,3)$ & $24(13,3)$ & & \\
$\quad$ Sí & & & & \\
Durante el tratamiento, ¿ingirió algún & & & & \\
tipo de sustancia alucinógena? & $63(73,3)$ & $145(80,6)$ & $1,66(1,36-2,20)$ & 0,000 \\
No & $23(26,7)$ & $35(19,4)$ & & \\
Sí & & & & \\
Durante el tratamiento, ¿fumó tabaco? & $54(62,8)$ & $132(73,3)$ & $1,61(1,35-2,06)$ & 0,000 \\
No & $32(37,2)$ & $48(26,7)$ & & \\
Sí & & & & \\
¿Cuántos cigarrillos fumó al día? & $7(21,9)$ & $38(79,2)$ & & \\
1 a 2 & $17(53,1)$ & $6(12,5)$ & $2,82(1,39-5,71)$ & 0,000 \\
3 a 4 & $8(25,0)$ & $4(8,39$ & $1,94(0,85-4,40)$ & 0,043 \\
5 a 10 & & &
\end{tabular}

OR: Odds ratio

Cuadro 4. Características de la población según la dimensión 'servicios de salud'

\begin{tabular}{|c|c|c|c|c|}
\hline Características & $\begin{array}{c}\text { Casos }(n=86) \\
n(\%)\end{array}$ & $\begin{array}{c}\text { Controles }(n=180) \\
n(\%)\end{array}$ & OR IC I5\% $_{9}$ & $\mathbf{p}$ \\
\hline \multicolumn{5}{|c|}{ Relación con el personal de salud } \\
\hline Buena & $16(18,6)$ & $111(61,7)$ & 1 & \\
\hline Regular & $48(55,8)$ & $48(26,7)$ & $1,55 \quad(1,25-1,92)$ & 0,000 \\
\hline $\begin{array}{l}\text { Mala } \\
\text { Explicación sobre }\end{array}$ & $22(25,6)$ & $3(1,7)$ & $6,12(2,11-17,74)$ & 0,000 \\
\hline No & $52(60,5)$ & $79(43,9)$ & $1,95 \quad(1,15-3,29)$ & 0,012 \\
\hline Sí & $34(39,5)$ & $101(56,1)$ & & \\
\hline \multicolumn{5}{|c|}{ Explicación sobre el tratamiento } \\
\hline No & $66(76,7)$ & $79(43,9)$ & $4,21 \quad(2,36-7,53)$ & 0,000 \\
\hline Sí & $20(23,3)$ & $101(56,1)$ & & \\
\hline \multicolumn{5}{|c|}{$\begin{array}{l}\text { ¿Considera adecuado el horario } \\
\text { de atención para la toma del } \\
\text { tratamiento? }\end{array}$} \\
\hline No & $70(81,4)$ & $44(24,4)$ & $13,52 \quad(7,12-5,66)$ & 0,000 \\
\hline \multicolumn{5}{|c|}{$\begin{array}{l}\text { ¿En algún momento dejó de } \\
\text { tomar medicamentos por falta de } \\
\text { personal? }\end{array}$} \\
\hline No & $33(38,4)$ & $141(78,3)$ & $0,17 \quad(0,09-0,30)$ & 0,000 \\
\hline Sí & $53(61,6)$ & $39(21,7)$ & & \\
\hline \multicolumn{5}{|c|}{$\begin{array}{l}\text { ¿Consideró cómodo el ambiente } \\
\text { donde recibió su tratamiento? }\end{array}$} \\
\hline No & $60(69,8)$ & $86(47,8)$ & $2,2 \quad(1,46-4,35)$ & 0,001 \\
\hline Sí & $26(30,2)$ & $95(52,2)$ & & \\
\hline \multicolumn{5}{|c|}{$\begin{array}{l}\text { ¿El personal de salud le } \\
\text { informó sobre sus resultados de } \\
\text { laboratorio? }\end{array}$} \\
\hline No & $73(84,9)$ & $42(23,3)$ & $18,45 \quad(9,31-6,55)$ & 0,000 \\
\hline Sí & $13(15,1)$ & $138(76,7)$ & & \\
\hline \multicolumn{5}{|c|}{$\begin{array}{l}\text { ¿Recibió atención oportuna } \\
\text { para sus consultas médicas y } \\
\text { exámenes de control? }\end{array}$} \\
\hline No & $75(87,2)$ & $47(26,1)$ & $19,29 \quad(9,44-9,43)$ & 0,000 \\
\hline Sí & $11(12,8)$ & $133(73,9)$ & & \\
\hline
\end{tabular}

OR: Odds ratio 
$\left(\mathrm{OR}=46,13 ; \mathrm{IC}_{95 \%}: 2,85-74,77 ; \mathrm{p}=0,007\right)$ (cuadro 5). La capacidad predicativa del modelo fue muy buena (93\%) según el área bajo la curva (Area Under the Curve, AUC) (figura 2).

Cuadro 5. Factores asociados con el abandono del tratamiento en pacientes con tuberculosis multirresistente según el modelo de regresión logística

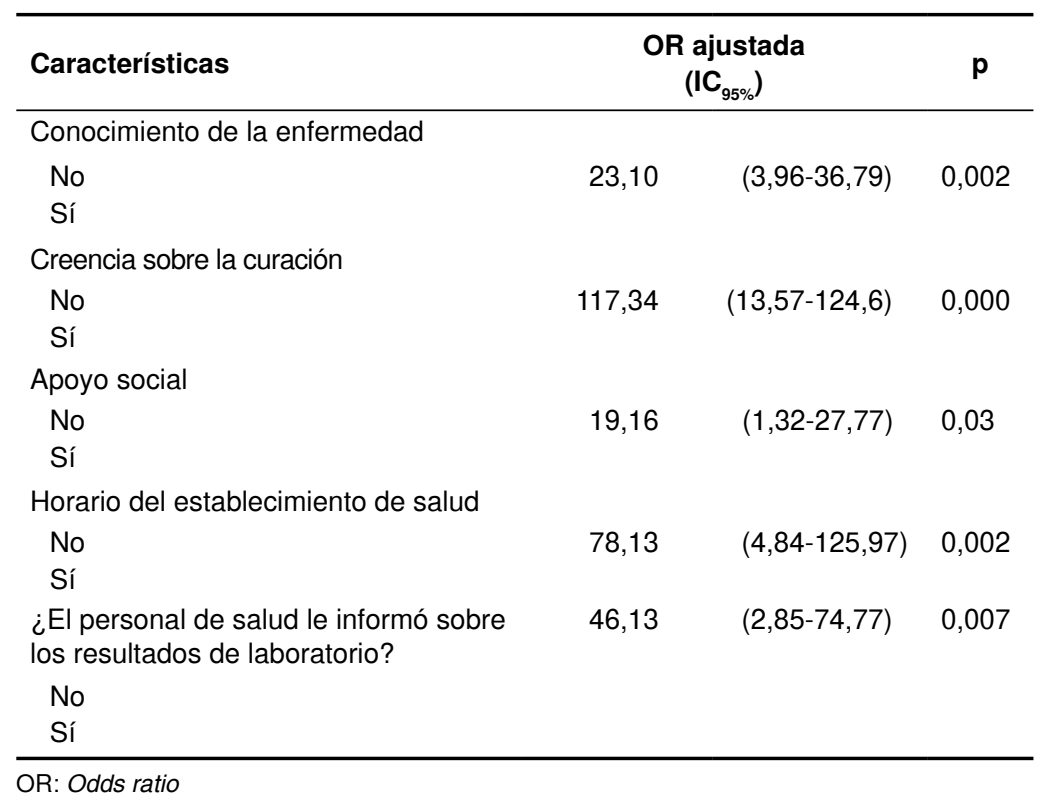

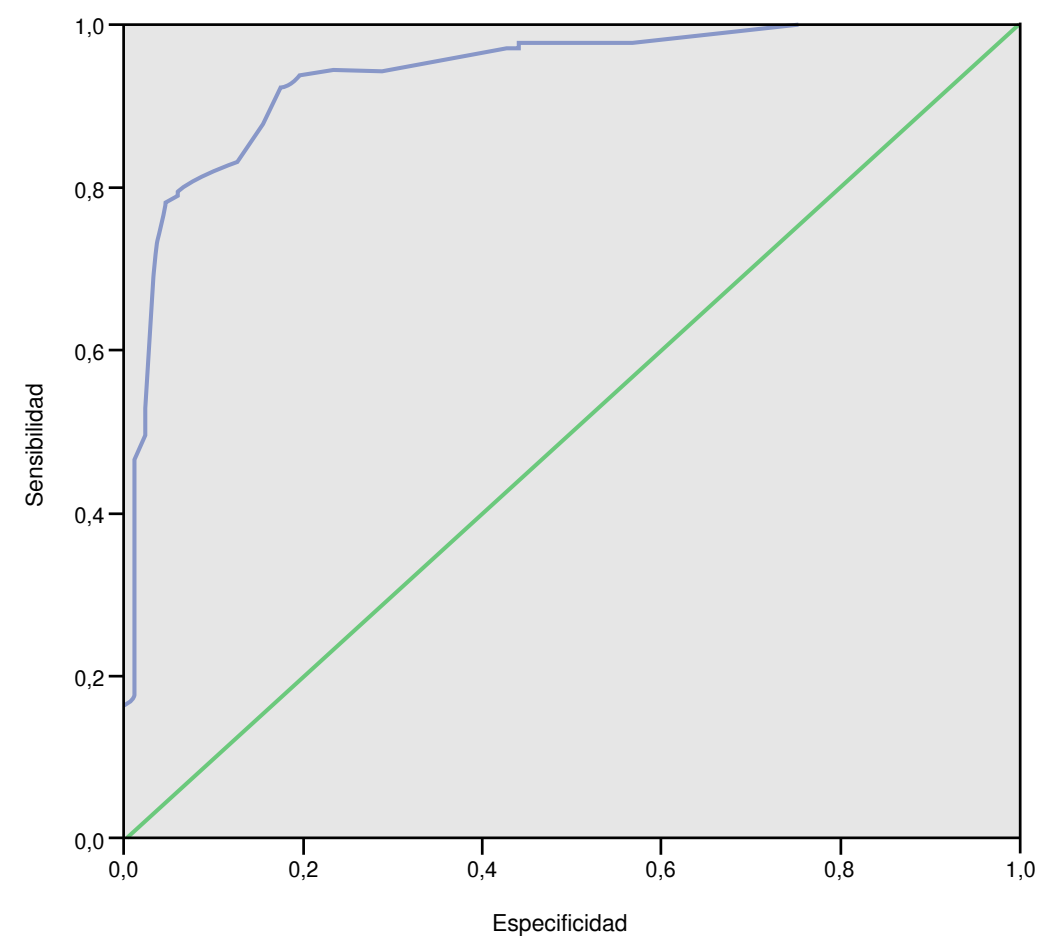

Figura 2. Curva del área bajo la curva del modelo multivariado de factores asociados con el abandono del tratamiento en pacientes con tuberculosis multirresistente 


\section{Discusión}

En los últimos 20 años se han hecho enormes esfuerzos por mejorar el tratamiento de la tuberculosis multirresistente, lo que ha implicado una importante inversión de recursos del Estado; sin embargo, ese esfuerzo resultará estéril si no se garantiza el cumplimiento del tratamiento. Conviene subrayar que, dadas sus múltiples dimensiones y causas, el abandono del tratamiento es un obstáculo que dificultaría mejorar la calidad de vida de la población.

La toma regular de los medicamentos antituberculosos en el contexto del programa ha demostrado sus beneficios, pero puede ocurrir que, por diversas circunstancias, el paciente no cumpla con el tratamiento o no respete su duración, lo que acrecienta la magnitud del problema que significa la enfermedad e implica un uso inadecuado de los recursos sanitarios y, más grave incluso, esa actitud puede llevar a la disminución del beneficio terapéutico de la curación y a limitar la interrupción de la cadena de transmisión, principales beneficios del tratamiento (11-15).

En esa perspectiva, el abandono del tratamiento conlleva resistencia secundaria o adquirida a medicamentos antituberculosos en pacientes que han seguido el tratamiento de forma irregular o que lo abandonan. Lo anterior favorece la aparición de cepas mutantes resistentes, tal como lo confirman los hallazgos de Gelmanova, et al. (15), en cuyo estudio se evidenció la asociación entre dicho incumplimiento y los malos resultados del tratamiento $\left(\mathrm{OR}=2,4 ; \mathrm{IC}_{95 \%}: 1,1-5,5\right)$, lo que significa un grave problema para el control de la enfermedad dado el incremento de la mortalidad y la diseminación de cepas de $M$. tuberculosis resistentes. Es evidente, entonces, que solo si el paciente toma regularmente el tratamiento, se obtienen los resultados esperados.

El análisis de la regresión logística multivariada en el presente estudio evidenció que el cumplimiento cabal del tratamiento en pacientes con tuberculosis multirresistente es un asunto complejo debido a un sinnúmero de condiciones: no tener conocimiento de la enfermedad, no creer en la curación, no contar con apoyo social, no considerar adecuado el horario de atención del establecimiento de salud y que los integrantes del equipo de atención no informen los resultados de laboratorio. Tales condiciones explican que alrededor del $30 \%$ de los pacientes que iniciaron el tratamiento para la tuberculosis multirresistente en la región del Callao, lo hubiera abandonado. En tales circunstancias, es difícil alcanzar las metas nacionales que, según los lineamientos de la OMS, incluyen reducir la mortalidad por tuberculosis en el $95 \%$ para el 2035 y la tasa de incidencia en el $90 \%$ en comparación con el año 2015 (1). Por tal razón, se requiere una participación activa de los servicios de salud, la familia, la comunidad y los pacientes para cerrar estas brechas.

Los factores de riesgo en este estudio concuerdan de manera general con lo hallado por Soza, et al. (16), quienes determinaron los siguientes para el abandono del tratamiento antituberculoso: ser de sexo masculino $(\mathrm{OR}=2,51$; $\left.\mathrm{IC}_{95 \%}: 1,63-3,94\right)$, residencia inestable o en la calle $\left(\mathrm{OR}=3,08 ; \mathrm{IC}_{95 \%}: 1,57-\right.$ $6,49)$, cambio de domicilio durante el tratamiento (OR=4,2; $\left.\mathrm{IC}_{95 \%}: 2,06-9,93\right)$, consumo de bebidas alcohólicas $\left(\mathrm{OR}=5,25 ; \mathrm{IC}_{95 \%}: 2,43-12,94\right)$, uso de drogas ilícitas (OR=5,25; IC $\left.{ }_{95 \%}: 2,43-12,94\right)$, dificultad de acceso a los servicios de salud ( $\left.\mathrm{OR}=2,64 ; \mathrm{IC}_{95 \%}: 1,39-5,29\right)$ y un concepto negativo de la atención recibida $\left(\mathrm{OR}=5,33 ; \mathrm{IC}_{95 \%}: 1,52-28,56\right)$. Es por ello que los autores plantean que en los servicios de salud deben adoptarse medidas para disminuir el riesgo de abandono y puntualizan la importancia de recuperar la participación social del sector salud mediante acciones comunitarias. 
Respecto a esto, Arrosi, et al. (17), encontraron que los pacientes con viviendas sin agua tenían tres veces más probabilidades de no cumplir el tratamiento (OR=2,8; $\left.\mathrm{IC}_{95 \%}, 1,1-6,9\right)$. Asimismo, los pacientes que tuvieron los controles en un hospital tenían tres veces más riesgo de no cumplir el tratamiento que quienes los tuvieron en centros de atención primaria $\left(\mathrm{OR}=3,2 ; \mathrm{IC}_{95 \%} 1,1-8,9\right)$. Según los investigadores, estos resultados permiten delinear un perfil del paciente en riesgo de no cumplir el tratamiento, que se caracteriza por estar en condiciones de pobreza y con dificultades de acceso a la atención en salud.

Por su parte, Sánchez, et al. (18) plantearon en su estudio cuantitativo y cualitativo que el abandono se relacionó con la poca tolerancia a los medicamentos, la eficacia del tratamiento y los factores sociales determinantes de las personas, así como a la deficiente educación brindada a los pacientes, por lo que recomendaron adaptarla a sus necesidades. Una mejor información, por ejemplo, sobre los efectos secundarios, podría ayudar al cumplimiento del tratamiento. Estos resultados concuerdan con el metaanálisis de Toczek, et al. (19), en el que concluyeron que la educación del paciente era una intervención eficaz para reducir el abandono del tratamiento.

En otro estudio, Maciel, et al. (20) encontraron que la falta de educación estaba muy relacionada con el abandono en los distritos de Rio de Janeiro, y recomendaron evaluarla teniendo en cuenta el contexto social en términos de su potencial para ampliar las oportunidades de trabajo, facilitar la movilidad social y reducir las desigualdades en la sociedad, dado que un buen nivel educativo está asociado con la buena salud, tal como lo demuestran otras investigaciones (21-25).

Los resultados de estos estudios permiten reconocer que no existe un perfil estándar del paciente que potencialmente abandonaría el tratamiento porque depende de cada realidad sociopolítica y demográfica, y de las múltiples causas y dimensiones que ello implica. Esto obliga a adelantar investigaciones operativas en las comunidades sobre este problema para conocer de primera mano cuáles son los factores de riesgo específicos.

El abandono del tratamiento es una situación clínica y operativa que hoy se considera una barrera para lograr el control de la tuberculosis en el mundo y es una de las áreas de acción preferentes para los programas nacionales del control de la enfermedad. Por ello, debe priorizarse y enfrentarse con estrategias diversas que contribuyan a incentivar una respuesta positiva de los afectados, sus familias y los servicios de salud, mediante una atención centrada en el paciente y basada en los ocho ejes temáticos planteados en la Primera Conferencia Ministerial Mundial de la OMS para poner fin a la tuberculosis en la era del desarrollo sostenible a través de una respuesta multisectorial:

"[...] cobertura universal de la prevención y atención de la tuberculosis; financiamiento sostenible y cobertura sanitaria universal y de protección social; respecto de la equidad, la ética y los derechos humanos; investigación e innovación científica; monitoreo y evaluación de los progresos; acción relativa a la resistencia a los antimicrobianos, seguridad sanitaria y tuberculosis multirresistente; aceleración de la respuesta a la tuberculosis y el VIH; por último, sinergias entre las respuestas a la tuberculosis y a las enfermedades no transmisibles [...]" $(17,26,27)$.

La salud es el resultado de una compleja interacción de factores: biología humana, ambiente, estilos de vida y organización de los servicios de salud que tienen un papel decisivo para entender la génesis y las consecuencias de enfermedades con muchos muchos factores externos influyentes, como 
la tuberculosis. Es en ese terreno que la prevención de la enfermedad y la promoción de la salud constituyen pilares fundamentales para enfrentar el abandono del tratamiento de la tuberculosis multirresistente. Para ello, es importante conocer el perfil de los potenciales candidatos a abandonar el tratamiento, explorando el comportamiento individual de las personas, sus creencias y sus actitudes y prácticas, en suma, su respuesta ante la enfermedad, pues influyen en la percepción de la prestación de los servicios y pueden contribuir al cumplimiento del tratamiento (28-30).

En el presente estudio, el análisis bivariado evidenció factores de riesgo que permiten caracterizar a los pacientes con tuberculosis multirresistente que abandonan el tratamiento: son personas de mediana edad, con un grado de instrucción medio, han tenido una hospitalización prolongada al inicio del tratamiento y durante el tratamiento no sintieron mejoría de la enfermedad. Además, son pacientes con comorbilidades como la diabetes mellitus. Podría tratarse de obreros, con un ingreso económico familiar equivalente al salario mínimo y que deben desplazarse largas distancias para llegar al establecimiento de salud a recibir su tratamiento. Además, no tienen conocimientos de la enfermedad, no creen en la mejoría, no reciben apoyo social o familiar y son consumidores de tabaco o drogas alucinógenas; mantienen una relación regular o mala con el personal del establecimiento de salud y no reciben explicación o información sobre la enfermedad, el tratamiento y los resultados de laboratorio; tampoco consideran adecuados el horario de atención, el tiempo de espera para recibir los medicamentos o las condiciones del ambiente donde reciben su tratamiento, y no reciben atención oportuna para las consultas médicas y exámenes de control. Es importante mencionar que los hallazgos del análisis bivariado no se evidenciaron totalmente en el estudio multivariado.

En otro estudio, Álvarez, et al. (31), reportaron que el desconocimiento de la enfermedad propicia la elección de diferentes alternativas, entre ellas, incumplir el tratamiento. Los servicios de salud y la inadecuada relación entre médico y paciente influyen en el retraso en el diagnóstico y la falta de cumplimiento del tratamiento, por lo que sugieren un programa de difusión sobre aspectos básicos de la enfermedad y el tratamiento.

Por otra parte, Deshmukh, et al. (32), manifestaron que el cumplimiento del tratamiento de la tuberculosis multirresistente es una tarea compleja tanto para los pacientes como para el sistema de salud, debido a su larga duración, a los factores socioeconómicos y personales de los pacientes y a sus estilos de vida, entre otros. Estos autores también resaltan algunos factores que contribuyen al éxito del tratamiento, como el apoyo social y la motivación, lo que se ha demostrado en varios países como Rusia e India (33-37), donde el apoyo del gobierno, de los voluntarios de la comunidad, así como de los vecinos, amigos y otros miembros de la comunidad, ha sido un factor importante para asegurar la finalización del tratamiento en estos pacientes.

Ahora bien, frente al abandono y la irregularidad del tratamiento, no puede dejar de mencionarse la importante incidencia de los responsables de las decisiones políticas, de la organización y la capacidad de prestación de servicios de las instituciones de la salud (tipo de establecimiento que ofrece la atención, tipo de proveedor o profesional que presta el servicio), de la evaluación del sistema de recuperación de pacientes, y de la disponibilidad de recursos financieros, humanos e insumos. Cuando estas instancias se enfrentan con problemas, ello se traduce en la debilidad en todo el sistema de salud, lo que, en últimas, contribuye al incumplimiento del tratamiento (38). 
En conclusión, se puede decir que la tuberculosis constituye un grave problema de salud pública en el mundo y que, aunque en la actualidad existen fármacos eficaces y potentes que podrían curar la enfermedad, esta ventaja se ve minimizada por el fenómeno del abandono del tratamiento. Se han descrito diversos factores que influyen en esta situación que, dadas las múltiples dimensiones que la afectan, debe ser afrontada desde diferentes perspectivas, lo que conlleva la necesidad de plantear aproximaciones más realistas al problema y establecer estrategias preventivas cuya relación costo efectividad y alto impacto contribuyan a que los afectados culminen exitosamente el tratamiento. En este sentido, no debe olvidarse que, una vez se presenta el abandono, las posibilidades de lograr que los pacientes retomen el tratamiento son muy bajas.

En este orden de ideas, debe fomentarse la aplicación de medidas preventivas en los ámbitos clínico, operacional, gerencial y de salud pública, para hacer el seguimiento de los casos que responden al perfil de riesgo ajustándose a las normas vigentes:

i. manejo clínico (historia clínica minuciosa y manejo multidisciplinario);

ii. manejo operativo (desarrollar los procedimientos para una adecuada asesoría y un acompañamiento eficaz del paciente por parte del personal de salud e integrantes de la comunidad);

iii. manejo gerencial (promover mecanismos para fortalecer la oferta de los servicios de salud: horarios adecuados, ambientes cómodos y asignación de recursos humanos);

iv. elaborar y ejecutar planes de información, educación y comunicación para los afectados;

v. impulsar el autocuidado de la salud, e

vi. intensificar las actividades de abogacía que contribuyan al desarrollo de políticas sanitarias orientadas hacia el trabajo coordinado entre los diversos sectores y la sociedad civil para lograr la fidelidad al tratamiento.

\section{Agradecimientos}

Al Hospital Daniel Alcides Carrión del Callao, Perú, por permitirnos la realización del estudio.

\section{Referencias}

1. World Health Organization - WHO. Global Tuberculosis Report, 2017. Fecha de consulta: 2 de mayo de 2018. Disponible en: http://apps.who.int/medicinedocs/es/d/Js23360en/

2. World Health Organization - WHO. Tuberculosis country profiles, 2016. Fecha de consulta: 19 de abril de 2018. Disponible en: http://www.who.int/tb/country/data/profiles/en/

3. Ministerio de Salud. Situación de la TBC en el Perú: normatividad, cobertura, casos y esquema de tratamiento. Lima: Ministerio de Salud; 2018. Fecha de consulta: 2 de mayo de 2018. Disponible en: ftp://ftp.minsa.gob.pe/sismed/ftp carga/REUNION\%20FORTALECIMIENTO\%20 SISMED\%20EN\%20EL\%20MARC0\%20DE\%20LAS\%20ESTRATEGIAS\%20DEL\%2012\%20 AL\%2015\%20MARZO\%202018/REUNION\%20TECNICA\%2012\%20_15\%20MARZO\%20 2018/REUNION\%20DEL\%2012 1503 18/Dia\%201/TBC.pdf

4. Bonilla C. Situación de la tuberculosis en el Perú. Acta Med Per. 2008;25:163-70.

5. Caminero J. Origen, presente y futuro de las resistencias en tuberculosis. Arch Bronconeumol. 2001;37:35-42. https://doi.org/10.1016/S0300-2896(01)75005-3

6. Rieder H. Bases epidemiológicas del control de la tuberculosis. Primera edición. Paris: Unión Internacional contra la Tuberculosis y Enfermedades Respiratorias; 1999. p. 172 
7. Farga V, Torres Z, Herrera M. Perfil del paciente con tuberculosis que abandona el tratamiento en Chile. Rev Chil Enf Respir. 2015;31:52-7. https://doi.org/10.4067/S0717-73482015000100008

8. Brito D, Sales M, Silva M, Silva S, Gomez L, Souza V. Fatores de adesao e risco de abandono ao tratamento. R Interd. 2015;8:169-79.

9. Culquí D, Munayco C, Grijalvac C, Cayla JA, Horna O, Kenedy A, et al. Factores asociados al abandono de tratamiento antituberculoso convencional en Perú. Arch Bronconeumol. 2012;48:150-5. https://doi.org/10.1016/j.arbres.2011.12.008

10. Ministerio de Salud. Norma técnica de salud para la atención integral de las personas afectadas por tuberculosis. Lima: MINSA; 2013.

11. Da Fonseca $P$, Silva $G$, Mendes $A$. Fatores asociados ao abandono do tratamento da tuberulose pulmonar no Maranhao, Brasil, no periodo de 2001 a 2010. Cad Saúde Pública. 2014;30:1745-54. https://doi.org/10.1590/0102-311X00124513

12. Calsin N, Horner B. Fatores associados ao abandon do tratamiento tuberculose: Uma revisao integrative. Texto Contexto Enferm. 2011;20:599-606.

13. Mitnick C, Bayona J, Palacios E, Shin S, Furin J, Alcántara F, et al. Community-based therapy for multidrug-resistant tuberculosis in Lima, Perú. N Engl J Med. 2003;348:119-28. https://doi.org/ 10.1056/NEJMoa022928

14. Comolet TM, Rakotomalala $\mathrm{R}$, Rajaonarioa $\mathrm{H}$. Factors determining compliance with tuberculosis treatment in an urban environment, Tamatave, Madagascar. Int J Tuberc Lung Dis. 1998;2:891-7.

15. Gelmanova I, Keshavjee S, Golubchikova VT. Barriers to successful tuberculosis treatment in Tomsk, Russian Federation: Non-adherence, default and the acquisition of multidrug resistance. Bull World Health Organ. 2007;85:703-11. https://doi.org/ 10.2471/BLT.06.038331

16. Soza NI, Pereira SM, Barreto ML. Abandono del tratamiento de la tuberculosis en Nicaragua: resultados de un estudio comparativo. Rev Panam Salud Pública. 2005;17:271-8.

17. Arrosi S, Herrero MB, Greco A, Ramos S. Factores asociados a la no adherencia al tratamiento de la tuberculosis: revisión de la literatura. Buenos Aires: CEDES; 2011. p. 37

18. Sánchez E, Marquer C, Kalon S, Qayyum S, Hayrapetyan A, Varaine F, et al. Reasons for defaulting from drug-resistant tuberculosis treatment in Armenia: A quantitative and qualitative study. Int J Tuberc Lung Dis. 2014;18:160-7. https://doi.org/ 10.5588/ijtld.13.0369

19. Toczek A, Cox H, du Cros P, Cooke G, Ford N. Strategies for reducing treatment default in drug-resistant tuberculosis: Systematic review and meta-analysis. Int J Tuberc Lung Dis. 2013;17:299-307. https://doi.org/10.5588/ijtld.12.0537

20. Maciel E, Amancio J, Castro D, Braga J. Social determinants of pulmonary tuberculosis treatment non-adherence in Rio de Janeiro, Brazil. PLoS One. 2018;13:e0190578. https://doi.org/10.1371/journal.pone.0190578

21. Sobral F, Fernanda A. Educacão para a competitividade ou para a cidadania social? São Paulo Em Perspect. 2000;14:3-11. https://doi.org/10.1590/S0102-88392000000100002

22. Vijay S, Kumar P, Chauhan LS, Vollepore BH, Kizhakkethil UP, Rao SG. Risk factors associated with default among new smear positive TB patients treated under DOTS in India. PLoS One. 2010;5:e10043. https://doi.org/0.1371/journal.pone.0010043

23. Dick J, Lombard C. Shared vision--a health education project designed to enhance adherence to anti-tuberculosis treatment. Int J Tuberc Lung Dis. 1997;1:181-6.

24. Comolet TM, Rakotomalala R, Rajaonarioa H. Factors determining compliance with tuberculosis treatment in an urban environment, Tamatave, Madagascar. Int J Tuberc Lung Dis. 1998;2:891-7.

25. Sabates $R$, Feinstein $L$. The role of education in the uptake of preventative health care: The case of cervical screening in Britain. Soc Sci Med. 2006;62:2998-3010. https://doi.org/10.1016/j.socscimed.2005.11.032

26. Farga V. Tuberculosis lo que hay que saber. Rev Chil Enf Respir. 2008;24:317-22. https://doi.org/10.4067/S0717-73482008000400008

27. World Health Organization - WHO. Primera Conferencia Ministerial Mundial. Fecha de consulta: 20 de octubre de 2018. Disponible en: http://www.who.int/tb/tb-factsheetconference-es.pdf?ua $=1$ 
28. Laframboise $\mathrm{HL}$. Health policy: Breaking the problem down into more manageable segments. Can Med Assoc J. 1973;108:388-91.

29. Lalonde M. A new perspective on the health of Canadians. Otawa: Minister of Supply and Services Canada; 1974.

30. Leimane V, Riekstina V, Holtz TH, Zarovska E, Skripconoka V, Thorpe L, et al. Clinical outcome of individualised treatment of multidrugresistant tuberculosis in Latvia: A retrospective cohort study. Lancet. 2005;365:318-26. https://doi.org/10.1016/S0140-6736(05)17786-1

31. Álvarez GC, Álvarez JF, Dorantes JE, Halperin D. Percepciones y prácticas relacionadas con la tuberculosis y la adherencia al tratamiento en Chiapas, México. Salud Pública Mex. 2000;42:520-8.

32. Deshmukh RD, Dhande DJ, Sachdeva KS, Sreenivas AN, Kumar AM, Parmar M. Social support a key factor for adherence to multidrug-resistant tuberculosis treatment. Indian $\mathrm{J}$ Tuberc. 2018;65:41-7. https://doi.org/10.1016/j.ijtb.2017.05.003

33. Barnhoorn F, Adriaanse $\mathrm{H}$. In search of factors responsible for noncompliance among tuberculosis patients in Wardha District, India. Soc Sci Med. 1992;34:291-306.

34. Jakubowiak WM, Bogorodskaya EM, Borisov SE, Danilova ID, Kourbatova EV. Risk factors associated with default among new pulmonary TB patients and social support in six Russian regions. Int J Tuberc Lung Dis. 2007;11:46-53.

35. Sengupta S, Pungrassami P, Balthip Q, Strauss R, Kasetjaroen Y, Chongsuvivatwong V, et al. Social impact of tuberculosis in southern Thailand: Views from patients, care providers and the community. Int J Tuberc Lung Dis. 2006;10:1008-12.

36. Ananthakrishnan R, Jeyaraj A, Palanil G, Sathiyasekaran B. Socio-economic impact of TB on patients registered within RNTCP and their families in the year 2007 in Chennai, India. Lung India. 2012;29:221-6. https://doi.org/10.4103/0970-2113.99103

37. Kaulagekar-Nagarkar A, Dhake D, Jha P. Perspective of tuberculosis patients on family support and care in rural Maharashtra. Indian J Tuberc. 2012;59:224-30.

38. Wendling A, Barbosa AP, Módena CM, Torres V. O abandono do tratamento da tuberculose sob a perspectiva dos gerentes de diferentes centros de saúde de Belo Horizonte-MG, Brasil. Text Context Enferm. 2012;21:77-85 https://doi.org/10.1590/S0104-07072012000100009 